\title{
Autophagy, replication stress and DNA synthesis, an intricate relationship
}

\author{
Francesco Cecconi $\mathbb{( i )}^{1,2}$
}

Received: 29 November 2019 / Accepted: 2 December 2019 / Published online: 3 January 2020

(c) The Author(s), under exclusive licence to ADMC Associazione Differenziamento e Morte Cellulare 2020

The cancer-relevance of autophagy is still debated, since this process is a very dynamic and context-dependent mechanism that can acts paradoxically both as tumor suppressor (by regulating DNA damage and oxidative stress), and as a tumor adaptive response (favouring malignant progression and chemoresistance) [1]. A typical cancer hallmark is genetic instability due to replication stresses (RS), DNA damage accumulation or a defective DNA repair machinery. Reflecting on the important role of autophagy in maintaining cellular homeostasis and integrity, it is not surprising that a link between autophagy and genome maintenance is found $[2,3]$, although yet not fully understood. A new study shines the spotlight on the kinetic role of autophagy in oncogene-induced tumorigenesis and as a key regulator of cancer cells recovering from RS/DNA damage; this work also offers a novel viewpoint over the molecular consequences that some common oncology drugs can undesirably drive [4].

In a recent issue by Vanzo et al. at least two important insights are reported [4]: (1) the authors bring novel proofs in favor of an autophagic role in tumor progression rather than suppression and (2), more unexpectedly, they uncover a novel role for autophagy in regulating DNA synthesis by stabilizing nucleotide pools and promoting genomic stability (Fig. 1).

By means of human tissue specimens from different stages of urinary bladder and prostate cancer, the group directed by Jiri Bartek found an induction of autophagy in

Francesco Cecconi

cecconi@ cancer.dk

1 Unit of Cell Stress and Survival, Center for Autophagy, Recycling and Disease (CARD), Danish Cancer Society Research Center, 2100 Copenhagen, Denmark

2 Department of Biology, University of Rome Tor Vergata and Department of Pediatric Hemato-Oncology and Cell and Gene therapy, IRCCS Bambino Gesù Children's Hospital, 00143 Rome, Italy early stages lesions when compared with normal tissues, without any changes during disease progression. In addition, although autophagy is thought to contribute towards tumor suppression by playing a role in ROS production, mitochondria accumulation and by inducing senescence [5], this work substantially challenges these concepts. Indeed, in autophagy-deficient conditions, Vanzo and coworkers detected increased mitochondrial mass and, in some models, enhanced oxidative respiration and glycolysis, as well as no oncogenic-dependent activation of cellular senescence. Hence, are mitochondria even more functional in ATG5/7 deficient cells? Although not directly explored, this hypothesis leads the authors to postulate that cells might counteract autophagy loss by upregulating their energy metabolism, in sharp contrast to what has been observed in murine models [6]. As a byside product, these findings may also ignite research on novel and still neglected aspects of mitophagy regulation such as the existing balance between mitochondria biogenesis and mitophagy upon general autophagy modulation or autophagosome impairment.

Furthermore, by using human cellular models, the authors got insight into the exact kinetics of autophagy induction in the context of oncogenic (H-RAS) and drugs (hydroxyurea, camptothecin)-dependent induction of RS and DNA damage response (DDR). They found that, in all models used, after the induction of RS, there is the activation of the cellular DDR signaling machinery, followed by autophagy activation at a later stage (Fig. 1). This is in line with the activation of both DNA damage checkpoints and autophagy in early human lesions analyzed by immunohistochemistry; however, at variance with autophagy, markers of DNA damage decline in advanced cancer. Collectively, these data support the intriguing possibility that autophagy is a positive response to RS-triggered DDR and is required for an effective and opportune recovery of the cells from RS.

With regards to the second issue, a more mechanistic view of the relationship between autophagy and RS in the cancer-context is provided in this article. Indeed, this study 


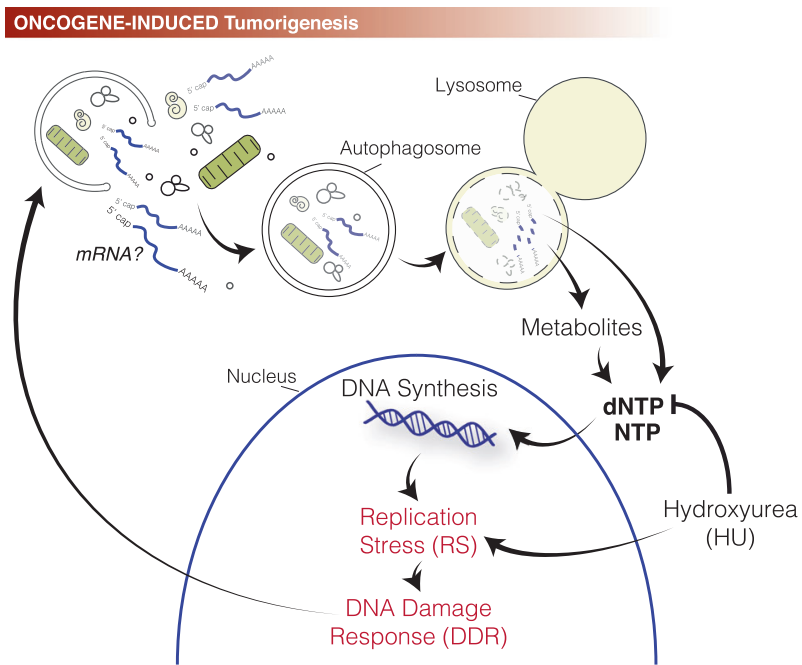

Fig. 1 The relationship among autophagy, DNA synthesis, and replication stress. Upon oncogene-induced tumorigenesis, autophagy activation provides the cancer cells with a stable pool of deoxyribonucleotides triphosphate (dNTPs), which sustain a high-rate of DNA synthesis, typical of cancer cells. This intense DNA replication causes Replication Stress (RS), which in turn triggers the DNA Damage Response (DDR). As a result of the DDR activation, autophagy is further enhanced, in order to help the cells to cope with the RS. In addition to oncogene-induced tumorigenesis, HydroxyUrea (HU) treatment can also cause RS, and trigger the above mentioned activation cascades. An intriguing possibility is the hypothetical relevance for autophagy in providing a ribonucleotides triphosphate (NTP) pool, derived by the autophagy-mediated degradation of cellular RNA (RNAphagy).

supports a role for autophagy in sustaining DNA synthesis in cancer cells by stabilizing dNTP pools. This may happen by inducing metabolite turnover that switches-on the molecular pathways leading to dNTP neosynthesis or through a novel (maybe selective) process that we may see as RNAphagy (Fig. 1). This notion is supported by a set of interesting experiments performed by the addition of exogenous deoxy-nucleotides that rescue the speed of replication fork elongation, thus mitigating the degree of RS and the RS-dependent DNA damage phenotype in autophagydeficient cells. Of note, previous work had shown that nucleoside/nucleotide metabolism during starvation relies on autophagy and that autophagy-dependent degradation of mRNA could provide metabolites to fuel nucleotide pools $[7,8]$.

In sum, this paper suggests that autophagy can support tumor progression and that cancer cells could exploit enhanced autophagy to survive and resist RS-inducing treatments, commonly used in oncology. Such a notion supports the importance of targeting autophagy to counteract its role in sustaining DNA synthesis and recovery from RS in cancer cells and encourages a more deep understanding of autophagy regulation before manipulating autophagy as an antitumor therapeutic strategy.

Acknowledgements The author is grateful to Francesca Nazio (Pediatric Hemato-Oncology and Cell and Gene therapy, IRCCS Bambino Gesù Children's Hospital, Rome) and Valentina Cianfanelli (Danish Cancer Society Research Center, Copenhagen) for discussion and help with text and Figure. I also acknowledge key fundings to Cecconi labs from the Danish Cancer Society (KBVU R72-A4408, R146-A9364), the Novo Nordisk Foundation (7559, 22544), the Lundbeckfonden (R233-2016-3360), the LEO Foundation (LF17024), "Associazione Italiana per la Ricerca sul Cancro" (AIRC IG-23543).

\section{Compliance with ethical standards}

Conflict of interest The author declares no conflict of interest.

Publisher's note Springer Nature remains neutral with regard to jurisdictional claims in published maps and institutional affiliations.

\section{References}

1. Singh SS, Vats S, Chia AY, Tan TZ, Deng S, Ong MS, et al. Dual role of autophagy in hallmarks of cancer. Oncogene. 2018; $37: 1142-58$.

2. Liu EY, Xu N, O'Prey J, Lao LY, Joshi S, Long JS, et al. Loss of autophagy causes a synthetic lethal deficiency in DNA repair. Proc Natl Acad Sci USA. 2015;112:773-8.

3. Wang Y, Zhu WG, \& Zhao Y. Autophagy substrate SQSTM1/p62 regulates chromatin ubiquitination during the DNA damage response. Autophagy. 2017;13:212-3.

4. Vanzo R, Bartkova J, Merchut-Maya JM, Hall A, Bouchal J, Dyrskjøt L, et al. Autophagy role(s) in response to oncogenes and DNA replication stress. Cell Death Differ. 2019. https://doi.org/10. 1038/s41418-019-0403-9.

5. Young AR, Narita M, Ferreira M, Kirschner K, Sadaie M, Darot JF, et al. Autophagy mediates the mitotic senescence transition. Genes Dev. 2009;23:798-803.

6. Galluzzi L, Pietrocola F, Bravo-San Pedro JM, Amaravadi RK, Baehrecke EH, Cecconi $\mathrm{F}$, et al. Autophagy in malignant transformation and cancer progression. EMBO J. 2015;34:856-80.

7. Medeiros TC, Thomas RL, Ghillebert R, \& Graef M. Autophagy balances mtDNA synthesis and degradation by DNA polymerase POLG during starvation. J cell Biol. 2018;217:1601-11.

8. Guo JY, Teng X, Laddha SV, Ma S, Van Nostrand SC, Yang Y. Autophagy provides metabolic substrates to maintain energy charge and nucleotide pools in Ras-driven lung cancer cells. Genes Dev. 2016;30:1704-17. 